\title{
TOPOLOGICAL CLASSIFICATION OF INFINITE DIMENSIONAL MANIFOLDS BY HOMOTOPY TYPE ${ }^{1}$
}

\author{
BY DAVID W. HENDERSON ${ }^{2}$ AND R. SCHORI
}

Communicated by Richard Anderson, August 4, 1969

1. Introduction. In this paper we prove that if $M$ and $N$ are connected paracompact manifolds modeled on a normed TVS, $F$, such that $F$ is homeomorphic $(\cong)$ to $F^{\omega}$ (countably infinite product of $F$ ), ${ }^{3}$ then $M$ and $N$ are homeomorphic if and only if they have the same homotopy type. We also prove that if $M$ and $N$ are connected paracompact manifolds modeled on a metrizable locally-convex (MLC) TVS, $F \cong F^{\omega}$, then each map $f: M \rightarrow N$ can be approximated by a closed embedding $g: M \rightarrow N$ and an open embedding $h: M \rightarrow N$ such that $f \sim g \sim h$ (homotopic). These and other results will be proved on the basis of results in recent, not yet published, papers written separately by the authors. See [5], [6], and [7]. These results already have been proved for separable Fréchet spaces by several authors, see [4] for references.

2. Theorems to quote. By manifold we will always mean a paracompact manifold. By TVS we mean a Hausdorff topological vector space.

S1. THEOREM [7]. If $M$ is a manifold modeled on a metrizable TVS, $F \cong F^{\omega}$, then $M \times F \cong M$.

Let $X$ and $Y$ be spaces, $\mathcal{u}$ be an open cover of $Y$, and $f, g: X \rightarrow Y$. Then $f$ and $g$ are said to be $\mathcal{u}$-approximate if for each $x \in X$ there is a

AMS Subject Classifications. Primary 5755; Secondary 5540, 5560, 5701.

Key Words and Phrases. Infinite-dimensional manifolds, homotopy type, topological vector space, microbundle, open embeddings, approximation by embeddings, Property $Z$, negligible.

${ }^{1}$ Research supported in part by NSF grants GP9397 and GP8637.

2 Alfred P. Sloan Fellow.

${ }^{3}$ Added in proof. This condition is satisfied by all infinite-dimensional Hilbert spaces, reflexive Banach spaces, and separable Fréchet spaces and is not known to be false for any Fréchet space. (See Bessaga and Kadec, On topological classification of (to appear).) 
$U \in \mathcal{U}$ that contains each of $f(x)$ and $g(x)$. If $C$ is a collection of functions from $X$ to $Y$, then $f$ is said to be approximated by members of $C$ if for each open cover $\mathcal{U}$ of $Y$, there exists $h \in C$ such that $f$ and $h$ are $\mathfrak{U}$-approximate.

S2. THEOREM [7]. Let $M$ be an open subset of a metrizable LCTVS, $F \cong F^{\omega}$. Then the projection map $p_{1}: M \times F \rightarrow M$ can be approximated by homeomorphisms $H: M \times F \rightarrow M$ such that $H \sim p_{1}$.

H1. ThEOREM [5]. A microbundle is trivial if

(a) its fiber is a TVS, $F$ such that $F \cong F^{\omega}$, and

(b) its base is a paracompact space with homotopy type of a simplicial (or CW-) complex.

H2. THEOREM [6]. If $M$ is a connected manifold modeled on a metrizable TVS, $F \cong F^{\omega}$, then $M$ can be embedded as a closed subset of $F$.

H3. THEOREM [6]. Let $M$ and $N$ be manifolds modeled on a MLCTVS, $F \cong F^{\omega}$. If $h: M \rightarrow N$ is a closed embedding, then there is an open embedding $g: M \times F \rightarrow N \times F$ such that $g(m, 0)=(h(m), 0)$ for each $m \in M$.

H4. THEOREM [6]. Let $N$ be a manifold modeled on a normed TVS, $F \cong F^{\omega}$, and let $X$ be an ANR (for metric spaces). If $f, g: X \rightarrow N$ are homotopic closed embeddings, then there is an invertible isotopy $h:(N \times F) \times I \rightarrow(N \times F) \times I$ such that $h(n, y, 0)=(n, y, 0)$ for each $n \in N$ and $y \in F$ and $h(f(x), 0,1)=(g(x), 0,1)$ for each $x \in X$.

$\mathrm{H} 4$ is a crucial step in proving

H5. THEOREM [6]. Let $M$ and $N$ be connected manifolds modeled on a normed TVS, $F \cong F^{\omega}$. If $f: M \rightarrow N$ is a homotopy equivalence, there exists a homeomorphism $h: M \times F \rightarrow N \times F$ such that $h \sim f \times$ id.

3. Theorems to prove. For each of the following theorems let $M$ and $N$ be connected paracompact manifolds modeled on a MLCTVS, $F \cong F^{\omega}$.

A. Theorem. The manifold $M$ can be embedded as an open subset of $F$.

CoRollary. The projection $p_{1}: M \times F \rightarrow M$ can be approximated by homeomorphisms $h: M \times F \rightarrow M$ such that $h \sim p_{1}$.

B. ThEOREM. Each map $f: M \rightarrow N$ can be approximated by closed embeddings $h_{1}: M \rightarrow N$ and open embeddings $h_{2}: M \rightarrow N$ such that $f \sim h_{1} \sim h_{2}$.

C. THEOREM. If $F$ is a normed TVS, then each homotopy equivalence between $M$ and $N$ is homotopic to a homeomorphism. 
Following R. D. Anderson we say that a subset $K$ of a space $X$ has Property $Z$ in $X$ if, for each nonempty, homotopically-trivial open set $U \subset X, U-K$ is nonempty and homotopically-trivial. The following theorem was proved for separable Fréchet manifolds in [1] and for special cases for nonseparable manifolds in [2].

D. Theorem. If $K$ is a closed set with Property $Z$ in $M$, then $K$ is negligible, that is, $M-K$ is homeomorphic to $M$. In fact, the homeomorphism is homotopic to the inclusion, $M-K \rightarrow M$.

4. Proofs. If $\mathcal{U}$ and $\vartheta$ are collections of subsets of a given set $X$, then to say that $v$ refines $\mathcal{u}$ means that each element of $v$ is contained in some element of $\mathcal{u}$. Denote this by $v<\mathcal{~}$. If $U \subset X$, let $\operatorname{St}(U, v)=U\{V \in v: U \cap V \neq \varnothing\}$ and let $\operatorname{St}(\mathcal{u}, v)=\{\operatorname{St}(U, v): U \in \mathcal{U}\}$.

Let $X$ and $Y$ be spaces, $\mathcal{u}$ and $\vartheta$ be open covers of $Y$, and $f, g, h$ : $X \rightarrow Y$. Then, $f$ and $g$ are $\mathcal{u}$-approximate if $\{\{f(x), g(x)\}: x \in X\}<\mathcal{u}$. Denote this by $\{f, g\}<\mathcal{U}$. If, in addition $\{g, h\}<\mathcal{V}$, then it follows that $\{f, h\}<\operatorname{St}(\mathcal{U}, \mathcal{v})$. Also denote $\left\{f^{-1}(U): U \in \mathcal{U}\right\}$ by $f^{-1}(\mathcal{U})$, $\{U \times F: U \in \mathcal{u}\}$ by $\mathcal{u} \times F$, and $\{\mathrm{Cl} U: U \in \mathcal{u}\}$ by $\mathrm{Cl} u$.

Proof of Theorem A. It follows immediately from $\mathrm{H} 2, \mathrm{H} 3$, and S1.

Proof of TheOREM B. Let $u$ be an open cover of $N$. We can assume that $N$ is an open subset of $F$ and that each element of $u$ is convex. By standard shrinking techniques we may find open covers $\mathcal{V}$ and $w$ of $N$ such that $\mathrm{Cl} w<v<\operatorname{St}(\operatorname{St}(v, w)$, $w)<\mathcal{~}$. By S2 take a homeomorphism $g^{\prime}: N \times F \rightarrow N$ that is "w-approximate to $p_{1}$. By H2 let $j$ be a closed embedding of $M$ into $F$. Then $h_{1}: M \rightarrow N$ defined by $h_{1}=g^{\prime} \cdot(f, j)$ is a closed embedding of $M$ into $N$ and $\left\{f, h_{1}\right\}<w$. Now apply H3 to $h_{1}: M \rightarrow N$ to obtain an open embedding $k: M \times F \rightarrow N \times F$. By S2 there is a homeomorphism $g: M \times F \rightarrow M$ such that $\left\{p_{1}, g\right\}$ $<h_{1}^{-1}(W)$. If $W \in W$, let $V(W) \in \mathcal{V}$ such that $\mathrm{Cl} W \subset V(W)$. Let $B=\left\{(x, y) \in M \times F\right.$ : if $x \in h_{1}^{-1}(W)$ for $W \in W$, then $k(x, y) \in V(W)$ $\times F\}$. Then $B$ is open in $M \times F$ and contains $M \times\{0\}$.

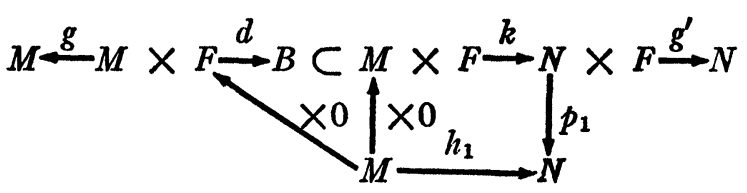

One can construct an open embedding $d: M \times F \rightarrow B$ (for example see [5, Lemma 1.2]) such that $p_{1}=p_{1} \circ d$ and the above diagram commutes.

Define $h_{2}: M \rightarrow N$ by $h_{2}=g^{\prime} \circ k \circ d \circ g^{-1}$. Since $\left\{g, p_{1}\right\}<h_{1}^{-1}(W)$ we 
have $\left\{g \circ g^{-1}, p_{1} \circ g^{-1}\right\}<h_{1}^{-1}(\mathscr{W})$ which is the same as $\left\{p_{1} \circ \times 0\right.$, $\left.p_{1} \circ g^{-1}\right\}<h_{1}^{-1}(w)$ since $p_{1} \circ \times 0=\mathrm{id}=g \circ g^{-1}$ and this means that $\left\{\times 0, g^{-1}\right\}<h_{1}^{-1}(\mathcal{W}) \times F$. Since $p_{1} \circ d=p_{1}$ we have $\left\{d \circ \times 0, d \circ g^{-1}\right\}$ $<h_{1}^{-1}(W) \times F$ and then by the definition of $B$ we have $\{k \circ d \circ \times 0$, $\left.k \circ d \circ g^{-1}\right\}<v \times F$ or equivalently $\left\{p_{1} \circ k \circ d \circ \times 0, p_{1} \circ k \circ d \circ g^{-1}\right\}$ $<\mathcal{V}$. Since $\left\{p_{1}, g^{\prime}\right\}<W$ we have $\left\{p_{1} \circ k \circ d \circ g^{-1}, g^{\prime} \circ k \circ d \circ g^{-1}\right\}<\mathscr{W}$ and hence $\left\{h_{1}, h_{2}\right\}<\operatorname{St}(\mathcal{V}, W)$ since $h_{1}=p_{1} \circ k \circ d \circ \times 0$ and $h_{2}$ $=g^{\prime} \circ k \circ d \circ g^{-1}$. We also have $\left\{f, h_{1}\right\}<w<\mathcal{U}$ and hence $\left\{f, h_{2}\right\}$ $<\operatorname{St}(\operatorname{St}(\mathcal{V}, \mathscr{W}), \mathscr{W})<\mathcal{U}$. Thus each of $h_{1}$ and $h_{2}$ is $\mathcal{U}$-approximate to $f$. Since each element of $u$ is convex, it is clear that $f \sim h_{1} \sim h_{2}$.

Proof of Theorem C. Let $f: M \rightarrow N$ be a homotopy equivalence. By $\mathrm{H} 5$ there exists a homeomorphism $h: M \times F \rightarrow N \times F$ such that $h \sim f \times$ id. By S2 there are homeomorphisms $g: M \times F \rightarrow M$ and $g^{\prime}: N \times F \rightarrow N$ such that $g \sim p_{1}$ and $g^{\prime} \sim p_{1}$. Then $g^{\prime} \circ h \circ g^{-1}$ is a homeomorphism of $M$ onto $N$ and $g^{\prime} \circ h \circ g^{-1} \sim p_{1} \circ h \circ g^{-1} \sim p_{1} \cdot(f \times$ id $) \circ g^{-1}$ $=f \circ p_{1} \circ g^{-1} \sim f \circ g \circ g^{-1} \sim f$.

Proof of Theorem D. It follows easily from Eells and Kuiper [3] that the inclusion $M-K \rightarrow M$ is a homotopy equivalence and thus by Theorem $\mathrm{C}$ the inclusion is homotopic to a homeomorphism.

\section{REFERENCES}

1. R. D. Anderson, D. W. Henderson and J. E. West, Negligible subsets of infinitedimensional manifolds, Compositio Math. 21 (1969), 143-150.

2. W. H. Cutler, Negligible subsets of nonseparable Hilbert manifolds, Proc. Amer. Math. Soc. (to appear).

3. J. Eells, Jr. and N. H. Kuiper, Homotopically negligible subsets of infinite-dimensional manifolds, Compositio Math. 21 (1969), 155-161.

4. D. W. Henderson, Infinite-dimensional manifolds are open subsets of Hilbert space, Bull. Amer. Math. Soc. 75 (1969), 759-762.

5. - Micro-bundles with infinite-dimensional fibers are trivial (to appear).

6. - Stable classification of infinite-dimensional manifolds by homotopy type (to appear).

7. R. Schori, Topological stability for infinite-dimensional manifolds (to appear).

Cornell University, Ithaca, New York 14850 and

Louisiana State University, Baton Rouge, Louisiana 70803 